\title{
Caracterização do Suporte Materno na Descoberta e Revelação do Abuso Sexual Infantil
}

\author{
Pedro Augusto Dias Baía ${ }^{1}$ \\ Tribunal de Justiça do Estado do Pará, Belém, Pará, Brasil \\ Celina Maria Colino Magalhães \\ Núcleo de Teoria e Pesquisa do Comportamento da Universidade Federal do Pará, \\ Belém, Pará, Brasil \\ Milene Maria Xavier Veloso \\ Programa de Pós-Graduação em Teoria e Pesquisa do Comportamento da Universidade \\ Federal do Pará, Belém, Pará, Brasil
}

\begin{abstract}
Resumo
O suporte materno pode funcionar como um moderador dos efeitos do abuso sexual no microssistema familiar das vítimas, maximizando as chances de ajustamento psicológico nestes contextos. Este estudo objetivou caracterizar o suporte materno de cinco mães de meninas vítimas de abuso sexual. Foram avaliadas as seguintes dimensões de suporte materno: acreditar na vítima, suporte emocional, e ação protetiva. Por meio das entrevistas, observou-se que a falta de credibilidade inicial das participantes em relação ao abuso sexual de suas filhas, não afetou as ações protetivas. Houve o relato de mudanças nas práticas maternas após a descoberta do abuso sexual. Discute-se a sobrecarga emocional destas cuidadoras, a manutenção do segredo acerca do abuso sexual e a importância das estratégias de intervenção familiar.
\end{abstract}

Palavras-chave: Revelação, abuso sexual, suporte materno.

\section{Characterization of Maternal Support in the Discovery and Disclosure of Child Sexual Abuse}

\begin{abstract}
The maternal support can act as a moderator of the effects of sexual abuse in the family microsystem victims, maximizing the chances of psychological adjustment in these contexts. This study aimed to characterize the maternal support of five mothers of sexually abused girls. The following dimensions of maternal support were considered: believe the victim, emotional support, and protective action. Through the interviews, observed that the initial lack of credibility of the participants in relation to the sexual abuse of their daughters, did not affect the protective actions. There was the report of changes in maternal practices after the discovery of sexual abuse. The emotional burden of these caregivers, the maintenance of the secrecy about sexual abuse, and the importance of family intervention strategies are discussed.
\end{abstract}

Keywords: Sexual abuse, disclosure, maternal support.

Endereço para correspondência: Núcleo de Teoria e Pesquisa do Comportamento, Universidade Federal do Pará, Av. Augusto Correa, s/n, Campus Universitário, Guamá, Belém, PA, Brasil 66075-900. E-mail: padbaia@ yahoo.com.br, celinaufpa@gmail.com e mileneveloso@hotmail.com

Agência de Financiamento: Fundação de Amparo à Pesquisa do Estado do Pará. 


\section{Caracterización de Apoyo Materno en el Descubrimiento y Develación de Abuso Sexual Infantil}

\section{Resumen}

El apoyo de la madre puede actuar como moderador de los efectos del abuso sexual en las víctimas de microsistemas familiares, maximizando las posibilidades de ajuste psicológico en estos contextos. Este estudio tuvo como objetivo caracterizar el apoyo materno de cinco madres de niñas víctimas de abuso sexual. Se consideraron las siguientes dimensiones del apoyo materno: creer en la víctima, el apoyo emocional y la acción protectora. A través de las entrevistas, se observó que la falta inicial de credibilidad de los participantes en relación con el abuso sexual de sus hijas, no afectó a las acciones de protección. Ha habido informes de cambios en las prácticas maternas después del descubrimiento de los abusos sexuales. Se discute la carga emocional de estos cuidadores, el mantenimiento del secreto sobre el abuso sexual y la importancia de las estrategias de intervención familiar.

Palabras clave: Abuso sexual, develación, apoyo materno.

A revelação do abuso sexual abrange duas dimensões: a revelação pela(s) própria(s) vítima(s), ou a detecção por outros indivíduos que não sejam o(a) agressor(a) e a(s) vitima(s). Constitui-se no primeiro caso em que o abuso sexual é descoberto ou divulgado (Nagel, Putnam, Noll, \& Trickett, 1997; Sepúlveda, 2010). Estudos empíricos e revisões bibliográficas descrevem a revelação como (a) intencional: quando ocorre a verbalização espontânea e não ambígua emitida pela própria criança/adolescente vítima; (b) indireta: quando alguns comentários feitos pela vítima eliciam questionamentos por parte do ouvinte, levando estas vítimas a revelarem; (c) estimulada: quando entrevistas investigativas ou técnicas de intervenção propiciam a ocorrência da revelação; (d) detecção por testemunha: situação na qual o abuso sexual foi diretamente testemunhado por uma segunda parte, a qual denunciou o abuso para o cuidador ou diretamente para uma autoridade; (e) acidental: a qual envolve situações nas quais uma segunda parte tomou conhecimento sobre o abuso a partir da observação de sinais físicos, mudanças comportamentais, ou alterações emocionais, por exemplo, levando ao questionamento da criança ou encaminhamento para um profissional (Alaggia, 2004; Campis, Hebden-Curtis, \& DeMaso, 1993; Collings, Griffith, \& Kumalo, 2005; Paine \& Hansen, 2002; Sepúlveda, 2010; Sgroi, Blick, $\&$ Porter, 1982; Sorensen \& Snow, 1991).
Relatar a experiência de abuso sexual envolve estratégias cognitivas e de linguagem, o que resultará em diferentes padrões de revelação dependendo da faixa etária das vítimas. Envolve também aspectos interativos existentes no microssistema familiar: entre a(s) vítima(s) e seu(sua) cuidador(a), entre a(s) vítima(s) e os(as) agressores(as). Complementarmente, aspectos de socialização, diferenças de gênero, e sentimentos de culpa ou vergonha, poderão incidir sobre as vítimas e o microssistema familiar, facilitando ou dificultando o processo de revelação (Alaggia, 2001; Collings et al., 2005; Paine $\&$ Hansen, 2002)

Neste sentido, a qualidade de suporte social recebida na época da descoberta do abuso sexual, está diretamente associada ao funcionamento psicológico das vítimas (ansiedade, depressão, auto imagem, relações familiares, por exemplo; Nagel et al., 1997; Pintello \& Zuravin, 2001; Tremblay, Herbert, \& Piche, 1999).

O suporte social é entendido como a existência ou a disponibilidade de pessoas nas quais o indivíduo poderá confiar. É composta por informações que conduzem o sujeito a acreditar que ele é amado e que as pessoas se preocupam com ele, o estimam e/ou valorizam, com sentimento de pertencimento a uma rede de comunicação e de deveres mútuos (Cobb, 1976; Sarason, Levine, Basham, \& Sarason, 1983). Entretanto, algumas consequências psicológicas advindas do 
abuso sexual, tais como o medo de intimidade e dificuldades para confiar, por exemplo, poderão também agir como obstáculos à ocorrência de suporte social. A intimidade e a confiança são componentes essenciais para formar a rede de relações sociais (Jonzon \& Lindblad, 2004).

No campo do suporte social, os estudos tem abordado o fenômeno do suporte materno, o qual é compreendido como um construto multidimensional, consistindo em: (a) credibilidade da mãe em relação à revelação da vítima; (b) ações protetivas no sentido de prevenir outras vitimizações da criança/adolescente vítima; e (c) reconhecimento e suporte ao possível stress da criança que se segue após o abuso e a revelação (Alaggia, 2002; Everson, Hunter, Runyon, Edelsohn, \& Coulter, 1989; Faller, 1998; Heriot, 1996; Sirles \& Franke, 1989; Smith et al., 2000).

As reações das mães diante da descoberta ou revelação do abuso sexual de seus(suas) filhos(as) pode ser crítico para determinar como estas vítimas irão se ajustar aos estressores advindos da experiência abusiva, da revelação, e se conseguirão adaptar-se inclusive ao processo de intervenção (Nagel et al., 1997; Pintello \& Zuravin, 2001; Smith et al., 2000). As vítimas que não percebem a existência de suporte materno no contexto familiar, poderão apresentar maiores chances de retratar a revelação, ainda que existam evidências robustas acerca do abuso sexual (Berliner \& Elliott, 1996).

Uma resposta considerada positiva ou suportiva tem sido frequentemente caracterizada pela demonstração de preocupação, empatia, e acreditar na vítima. Por outro lado, uma reposta negativa e não suportiva é caracteriza pela culpabilização da vítima, negação ou minimização do evento abusivo, e inclusive o abandono da vítima pela genitora em alguns casos (Lamb \& Edgar-Smith, 1994)

Em uma pesquisa com 10 mães de meninas vítimas de abuso sexual, Santos e Dell'Aglio (2009) observaram que as respostas das mães quanto à notícia de abuso sexual de suas filhas centravam-se em duas dimensões: credibilidade e ação. As autoras sugerem que a credibilidade refere-se ao fato destas mães acreditarem ou não na veracidade do relato de abuso sexual de suas filhas, enquanto que a dimensão da ação está relacionada a ter ou não a iniciativa de realizar a notificação. Esta pesquisa traz alguns dados similares aos de Bolen e Lamb (2004), no qual se observou que a ambivalência e o suporte materno podem ser constructos independentes.

A presença ou não de suporte materno está associado, principalmente, aos seguintes fatores: o tipo de relacionamento das mães com os agressores, a história materna de abuso sexual, idade das vítimas, e o gênero das vítimas (Cyr et al., 2003; DeYoung, 1994; Elliott \& Carnes, 2001; Heriot, 1996; Pintello \& Zuravin, 2001; Sirles \& Franke, 1989).

As informações de caráter verbal ou comportamental apresentadas pelas vítimas se constituem nas fontes mais comuns de informação utilizadas pelas mães para acreditarem ou não na ocorrência da violência (Lawson \& Chaffin, 1992; Plummer, 2006). As percepções, interpretações e ações maternas podem explicar se estas cuidadoras irão ou não negligenciar seus(suas) filhos(as). Assim, o processamento da informação para as cuidadoras se inicia com um sinal de que seus(suas) filhos(as) estão com necessidades não atendidas. Se estas cuidadoras não percebem tais necessidades, não haverá processamento das informações e consequentemente, nenhuma ação protetiva poderá ocorrer. Em contextos de abuso sexual, as cuidadoras poderão receber informações acerca da violência por meio de diferentes fontes que sinalizam tais necessidades de proteção. Tais fontes poderão consistir, por exemplo, em uma denúncia formal feita pelas autoridades, a revelação feita pela própria vítima, a observação de sinais físicos/comportamentais, por exemplo (Crittenden, 1993).

A revelação do abuso sexual e sua consequente descoberta pelo meio familiar e/ou social $\mathrm{da}(\mathrm{s})$ vítima(s) representam um contexto de crise, com a eclosão de conflitos e sentimentos negativos no meio familiar (Levenson, Tewksbury, \& DiGiorgio-Miller, 2012). O sentimento de vergonha tem sido tratado na literatura como um fator comum a determinados grupos culturais, e que tem um impacto evidente na maneira como a vítima e seu próprio meio familiar irá se comportar (Boakye, 2009; Fontes, 2007). De acordo 
com Boakye (2009), o "problema da vergonha coletiva" (collective shame problem - CSP) é uma tendência apresentada por indivíduos de um grupo particular (família, clã ou linhagem), para sentirem ou expressarem um forte sentimento de embaraço após uma atitude ou comportamento indesejado emitido por um membro do grupo. Tais atitudes ou comportamentos são considerados pelos indivíduos com potencial para ferir ou ameaçar a reputação do grupo como um todo. Este autor propõe que em um ambiente cultural onde o senso de identidade e honra do indivíduo são fortemente ligadas à família, a tendência para apresentar uma proteção indiscriminada aos interesses coletivos será maior, em detrimento ao interesse individual (vítimas de abuso sexual intrafamiliar, por exemplo). Com isto, Boakye (2009) argumenta que a revelação do abuso sexual e a posterior denúncia aos órgãos competentes poderão ser mais difíceis nas sociedades com características coletivistas do que naquelas predominantemente individualistas (Boakye, 2009).

Assim, este estudo objetivou caracterizar o suporte materno de mães de crianças e adolescentes vítimas de abuso sexual, a partir da realização de entrevistas semiestruturadas com questões relacionadas às seguintes dimensões: acreditar na criança/adolescente vítima, suporte emocional e ação protetiva. A literatura especializada situa estas três dimensões como domínios importantes para a adaptação das vítimas após a revelação/descoberta do abuso sexual, bem como se constituem em eixos norteadores para a intervenção psicossocial e legal (Alaggia, 2002; Coohey \& O'Leary, 2008; Cyr et al., 2003; Pintello \& Zuravin, 2001; Smith et al., 2000).

\section{Método}

\section{Participantes}

Participaram deste estudo cinco mães de meninas vítimas de abuso sexual intrafamiliar (um caso) e extrafamiliar (quatro casos). As mães e suas filhas estavam recebendo atendimento psicossocial em um Centro de Referência Especializado em Assistência Social (CREAS), localizado no município de Abaetetuba (Pará). Estas mães possuíam idades variando de 24 a 50 anos, com a escolaridade do ensino fundamental ao médio completo.

O município de Abaetetuba faz parte da Microrregião de Cametá e Mesorregião Paraense, com uma área aproximada de $1223,47 \mathrm{~km}^{2} \mathrm{e}$ população de 141,100 hab., com uma densidade em torno de 115,29 hab $/ \mathrm{km}^{2}$. Está distante cerca de 101,5 km da capital Belém. A atividade econômica predominante é o setor primário e terciário. O Índice de Desenvolvimento Humano Municipal (IDHM) de Abaetetuba foi de 0,628, em 2010, o que o situa na faixa de IDHM Médio (IDHM entre 0,6 e 0,699; Programa das Nações Unidas para o Desenvolvimento, Instituto de Pesquisa Econômica Aplicada, \& Fundação João Pinheiro, 2013)

Foram utilizados os seguintes critérios de inclusão das participantes na amostra: (a) mães com idades acima de 18 anos; (b) que estavam coabitando com as vítimas quando ocorreu a descoberta do abuso sexual; (c) mães que não foram denunciadas como agressoras sexuais.

\section{Instrumentos/Materiais}

Foram realizadas entrevistas individuais semiestruturadas com o objetivo de conhecer o suporte materno de genitoras após a descoberta do abuso sexual sofrido pelas suas filhas. As entrevistas foram realizadas nas dependências da instituição com duração média de uma hora cada. A entrevista semiestruturada foi elaborada baseando-se na literatura (Alaggia, 2002; Coohey \& O'Leary, 2008; Cyr et al., 2003; Pintello \& Zuravin, 2001; Smith et al., 2000), sendo formuladas questões associadas as seguintes dimensões do suporte materno: acreditar na vitima (o grau de credibilidade da mãe em relação à descrição sobre o abuso feita pela própria vítima), suporte emocional (empatia pelo estresse experimentado pela vítima, ausência de rejeição ou emoções negativas), e ação protetiva (manter a criança segura de possíveis abusos futuros e realizar denúncias)

\section{Procedimentos}

Em uma primeira fase, o projeto foi submetido para avaliação do Comitê de Ética do Núcleo de Medicina Tropical da Universidade 
Federal do Pará, e recebeu parecer favorável à realização (CAAE: 03037412.7.0000.5172, parecer $\left.\mathrm{n}^{\circ} 87.828\right)$. O estudo seguiu as normas da Resolução 466/2012 do Conselho Nacional de Saúde (2012), que regulamenta as normas aplicadas a pesquisas que envolvem, diretamente ou indiretamente, seres humanos. Em uma segunda fase, o pesquisador reuniu-se com a equipe técnica do CREAS-Abaetetuba, objetivando explicar o projeto e os procedimentos a serem realizados. Foi disponibilizado o Termo de Concordância da Instituição. Considerando os aspectos éticos da confidencialidade, os profissionais técnicos do CREAS informaram sobre a realização da pesquisa às mães que estavam em atendimento no CREAS. Na terceira fase, as participantes que aceitaram participar da pesquisa compareceram ao CREAS em hora e data agendadas. O pesquisador procedia a leitura do Termo de Consentimento Livre e Esclarecido, e após a assinatura deste documento se iniciavam as entrevistas, com duração média de uma hora para cada participante.

\section{Análise dos Dados}

Os dados oriundos das entrevistas realizadas com as mães foram tratados a partir da análise de conteúdo (Bardin, 1977/2006), averiguando-se as seguintes dimensões de suporte materno: acreditar na vítima, suporte emocional, e ação protetiva.

\section{Resultados}

Foram entrevistadas cinco mães de meninas vítimas de abuso sexual, com idades variando de 24 a 50 anos. A quantidade da prole de cada genitora variou de um a três filhos(as). Todas as mães realizaram a denúncia do abuso sexual de suas filhas ao conselho tutelar, delegacia de policia ou CREAS da região.

Através da Tabela 1 observa-se que a idade das vítimas variou de quatro a 13 anos. Todos os agressores tinham idades acima de 18 anos, e o abuso sexual foi predominantemente extrafamiliar $(n=4)$, denunciados pelas genitoras em sua maioria $(n=5)$. Acerca da revelação do abuso sexual, registrou-se um caso no qual a genitora foi testemunha do abuso sexual (detecção por testemunha), três casos nos quais as genitoras observaram mudanças comportamentais/físicas e descobriram o abuso sexual (detecção acidental), e um caso envolvendo diretamente revelação intencional.

Tabela 1

Dados Sóciodemográficos das Participantes e Informações sobre o Abuso Sexual

\begin{tabular}{cccccccc}
\hline Participante & Idade & Situação Profissional & $\begin{array}{c}\text { Situação } \\
\text { conjugal }\end{array}$ & $\begin{array}{c}\mathrm{N}^{\mathrm{o}} \text { de } \\
\text { filhos }\end{array}$ & $\begin{array}{c}\text { Idade da } \\
\text { vítima }\end{array}$ & $\begin{array}{c}\text { Agressor(a) } \\
\text { agressor }\end{array}$ \\
\hline 1 & 24 & Vendedora & Solteira & 01 & 4 & Extrafamiliar & 50 anos \\
2 & 28 & Operadora de caixa & Convivente & 01 & 8 & Intrafamiliar & +18 anos \\
3 & 37 & Funcionária pública & Casada & 03 & 11 & Extrafamiliar & +18 anos \\
4 & 42 & Do lar & Separada & 02 & 6 & Extrafamiliar & +18 anos \\
5 & 50 & Funcionária pública & Separada & 02 & 13 & Extrafamiliar & 21 anos \\
\hline
\end{tabular}

\section{Dimensões do Suporte Materno}

Acreditar na Vitima. A partir das entrevistas realizadas, observou-se que todas as genitoras afirmaram, inicialmente, ter acreditado no relato de suas filhas na época da descoberta do abuso sexual. Posteriormente, verificou-se que esta credibilidade não foi imediata, pois as cuidadoras buscaram indícios comportamentais ou externos para reforçar e confirmar os relatos.

Estas participantes apontaram como fatores importantes para a credibilidade: (a) mudanças comportamentais por parte das vítimas: “ $A$ 
mudança comportamental foi muito grande. Eu senti, ela não queria ir pra escola. Isso aí está estranho" (Participante 3). (b) a cena testemunhada: "Ninguém me contou, eu vi" (Participante 1). (c) a confirmação do abuso sexual por profissionais especializados: "Eu só consegui acreditar quando eu fui em B... e ela contou detalhadamente pra psicóloga" (Participante 5).

Os relatos iniciais das vítimas não foram suficientes para suprir as dúvidas das genitoras, levando-as a se utilizar de questionamentos após a descoberta do abuso sexual. Verificou-se que tais questionamentos estiveram centrados principalmente em: (a) identificação do agressor: "Minha filha, me fala quem foi... como ele era!?" (Participante 1). (b) verificar se houve penetração e/ou violências físicas diversas: "Aonde ele te tocou?" (Participante 2). (c) histórico de outras experiências abusivas: "isso que ele fez aconteceu outras vezes?" (Participante 5).

Observou-se também que algumas cuidadoras remeteram-se às práticas maternas anteriores ao abuso sexual como forma de justificar e garantir que acreditaram no relato de suas filhas, independentemente se o abuso foi descoberto ou revelado pelas próprias crianças:

Porque eu compro uns livros pra ela, e toda noite eu leio. E eu falo pra ela: É feio mentir, papai do céu fica triste. E eu nunca senti que ela minta pra mim. Tudo que acontece ela fala pra mim. Ás vezes eu fico em dúvida e pergunto pra professora. E por ai eu tiro, que ela não mentiu. (Participante 1)

"Eu criei elas de um jeito que tudo o que ela fizesse, ela ia me contar. Acho que depende da educação que eu dei. Ela sabia que ia ter apoio" (Participante 5).

Suporte Emocional. A partir das entrevistas realizadas, observou-se que as cuidadoras demonstraram empatia em relação às suas filhas, presença de sentimentos de tristeza, raiva em relação ao agressor, e o reconhecimento de que a situação abusiva acarretaria dificuldades a nível psicológico e físico para as vítimas.

"Não acredito que ela poderia ter feito algo para impedir, pois ela é uma criança indefesa" (Participante 4).
"Você vai me ajudar, e eu vou te ajudar. A gente precisa se unir e colocar ele na cadeia" (Participante 3).

"Não achei que ela 'tivesse' mentindo. Eu vi no semblante dela, ela falava, ela chorava" (Participante 5).

"Porque ele cometeu um crime grave. Pra mim ela era uma criança" (Participante 2).

"Depois do que aconteceu ela ia ter problemas, eu pensei, por isso logo pedi ajuda" (Participante 1).

Verificou-se também que pelo menos duas participantes questionaram as vítimas acerca da possibilidade destas terem evitado o contato sexual com o agressor:

foi a primeira coisa que eu falei: "Foi quatro vezes, por que minha filha? Se na primeira vez ele já te levou pra um lugar inadequado, porque ainda teve outras vezes... por que você não refletiu, Porque você deixou acontecer?". (Participante 3 )

"Como eu falei, quando a gente não conhece uma pessoa, a gente não vai. Se tu 'tava' conversando com a tua colega, por que 'tu foi'?" (Participante 5).

Ação Protetiva. Os relatos das cuidadoras e as informações de cada caso sugerem que as principais atitudes tomadas foram: (a) averiguar a situação física das vítimas (buscar indícios de penetração e/quaisquer outras violências físicas); (b) colocar a vítima em segurança junto a um familiar; (c) procurar o conselho tutelar ou a delegacia da localidade; e (d) comparecer ao CREAS. Todas as participantes entrevistadas demonstraram certeza de que suas atitudes, após a descoberta do abuso sexual de suas filhas, foram as mais adequadas.

"Aí eu chamei a avó dela e pedi pra ficar com ela enquanto eu ia no Conselho Tutelar" (Participante 1).

"Eu logo fui examinar ela, olhei as partes intimas dela pra ver o que tinha acontecido..." (Participante 3).

"depois que eu fiquei sabendo, eu fui fazer o Boletim de coorrência, aí já veio o Conselho Tutelar e depois eu levei ela no CREAS" (Participante 4). 


\section{Discussão}

As dimensões de suporte materno tratadas na literatura mostraram-se presentes na amostra estudada. Inicialmente, as genitoras entrevistadas relataram credibilidade em relação ao relato de abuso sexual de suas filhas e suporte emocional à experiência traumática. Posteriormente, as genitoras afirmaram que necessitaram de indícios complementares para acreditarem nos relatos das filhas. E desta forma, utilizaram-se de questionamentos às vítimas, com o objetivo de conhecer a identidade do agressor, verificar se houve penetração e/ou violências físicas diversas, e o histórico de outras experiências abusivas. Tais questionamentos ampliaram as fontes de informação para as genitoras, contribuindo inclusive para a implementação das ações de proteção. Em três casos analisados, foram as informações de caráter físico e verbal das vítimas que se constituíram nas fontes de informação para que as genitoras determinassem se o abuso tinha ou não ocorrido. Tais resultados também foram observados em estudos prévios (Coohey \& O'Leary, 2008; Crittenden, 1993; Lawson \& Chaffin, 1992; Plummer, 2006).

A amostra do presente estudo foi composta em sua maioria por casos de abuso sexual extrafamiliar, os quais são associados a suporte materno mais adequado em comparação a contextos de abuso intrafamiliar (Cyr et al., 2003; Elliott \& Carnes, 2001; Sirles \& Franke, 1989). É possível que em decorrência deste aspecto, todas as genitoras entrevistadas tenham apresentado um suporte materno caracterizado pela credibilidade em relação ao relato das vítimas. Observou-se também a implementação de ações de proteção (após a descoberta do abuso sexual, buscaram afastar as vítimas do contato com o agressor, mantiveram-nas sob a sua supervisão ou sob os cuidados de um familiar responsável, realizaram a denúncia contra os agressores, e se engajaram no acompanhamento psicossocial às vítimas). Quando não estão em relacionamento afetivo/ sexual com os agressores na época do abuso sexual, as mães poderão vivenciar menos conflitos para conciliar o seu papel de cuidadora em relação à vítima e o dever em denunciar o agressor (Pintello \& Zuravin, 2001).
Por outro lado, ainda que tenham manifestado tal credibilidade à revelação do abuso sexual de suas filhas e utilizado estratégias de proteção, tais participantes relataram a manutenção do segredo em torno do ato abusivo. Ou seja, algumas destas mães não haviam divulgado aos outros membros familiares as informações acerca do abuso sexual de suas filhas. Conflitos familiares e o sentimento de vergonha foram apontados como os principais motivos para que estas mães não compartilhassem a informação, o que pode sugerir dificuldades ao nível da intimidade e confiança (Jonzon \& Lindblad, 2004), além da incidência do fator vergonha ao nível individual e cultural (Boakye, 2009; Fontes, 2007). Estas mães mencionaram o medo de serem julgadas pela comunidade na qual estavam inseridas, ou o que de ruim poderia acontecer caso a comunidade tomasse conhecimento do fato ocorrido com as suas filhas.

Uma problemática advinda desta manutenção do segredo em torno do abuso sexual extrafamiliar é a não inserção de outros familiares (genitores, irmãos, tios, por exemplo) nos procedimentos psicossociais. Consequentemente, a rede de suporte social se cristaliza unicamente no nível do suporte materno, levando a uma sobrecarga psicológica e emocional às mães.

De outra forma, ainda que a descoberta do abuso sexual gere dificuldades ao nível pessoal e social, três participantes entrevistadas afirmaram que, após tomarem conhecimento acerca do abuso sexual, buscaram acentuar suas práticas maternas no campo da supervisão comportamental, diálogo e estabelecimento da confiança na relação com a vítima. Estes dados se coadunam a outros estudos (Plummer, 2006; Santos \& Dell'Aglio, 2013), constando que algumas genitoras perceberam mudanças positivas (mais protetivas, próximas e atentas) no relacionamento com a prole após a descoberta do abuso sexual.

\section{Considerações Finais}

O estudo aponta elementos relevantes sobre as questões do suporte materno dado a crianças e adolescentes vítimas de abuso sexual. A incidência de questionamentos por parte das mães, 
direcionados às vítimas com o objetivo de obter maiores informações sobre o abuso sexual, não alterou as ações protetivas implementadas. Entretanto, as participantes relataram o sentimento de vergonha como um fator que as impediu de compartilhar sobre o abuso sexual aos outros familiares (genitor das vítimas, irmãos, tios, por exemplo).

Sugere-se que as instituições de atendimentos especializados às vítimas de abuso sexual integrem em seus protocolos de atendimento, inclusive nas etapas iniciais de anamnese e acolhimento, a avaliação acerca do suporte materno. Esta avaliação objetivará, por exemplo: (a) diagnosticar o nível de suporte materno; (b) verificar a organização do microssistema familiar das vítimas: vínculo, estrutura, relação genitor e genitora, instabilidades relacionais advindas da descoberta do abuso sexual; (c) reforçar a importância do suporte familiar no processo de acompanhamento psicossocial às vítimas. Necessário também é a realização de pesquisas buscando avaliar de que formas os estilos parentais utilizados no microssistema familiar estão correlacionados ao suporte materno em contextos de descoberta e revelação do abuso sexual, como forma de propor estratégias de intervenção em contextos familiares com déficit no suporte familiar.

A presente pesquisa se mostrou limitada por não utilizar um instrumental para acessar os fatores culturais presentes no contexto estudado. Tal amostra possui características próprias sob diversos aspectos, os quais podem se refletir na maneira como o abuso foi descoberto e inclusive o acesso das vítimas aos serviços de atendimento. Outra limitação constatada foi a predominância de casos de abuso sexual extrafamiliar. Os resultados obtidos devem ser observados levando em consideração as dinâmicas deste tipo de abuso, nas quais geralmente o agressor não habita o mesmo lar das vítimas, ou não se constitui em um companheiro afetivo das genitoras. Estudos futuros poderiam utilizar-se de uma metodologia que pudesse comparar o suporte materno em contextos de abuso sexual intrafamiliar e extrafamiliar, com a ampliação da amostra.

\section{Referências}

Alaggia, R. (2001). Cultural and religious influences in maternal response to intrafamilial child sexual abuse: Charting new territory for research and treatment. Journal of Child Sexual Abuse, 10(2), 41-60. doi:10.1300/J070v10n0203

Alaggia, R. (2002). Balancing acts: Re-conceptualizing support in maternal response to intrafamilial child sexual abuse. Clinical Social Work Journal, 30(1), 41-56. doi:10.1023/A:1014274311428

Alaggia, R. (2004). Many ways of telling: Expanding conceptualizations. Child Abuse \& Neglect, 28, 1213-1227. doi:10.1016/j.chiabu.2004.03.016

Bardin, L. (2006). Análise de conteúdo (L. de A. Rego \& A. Pinheiro, Trads.). Lisboa, Portugal: Edições 70. (Original publicado em 1977)

Berliner, L., \& Elliott, D. M. (1996). Sexual abuse of children. In J. E. B. Myers, L. Berliner, J. Briere, C. T. Hendrix, C. Jenny, \& T. A. Reid (Eds.), The APSAC Handbook on child maltreatment ( $2^{\text {nd }}$ ed., pp. 55-78). Thousand Oaks, CA: Sage.

Boakye, K. E. (2009). Culture and nondisclosure of child sexual abuse in Ghana: A theoretical and empirical exploration. Law \& Social Inquiry, 34(4), 951-979. doi:10.1111/j.17474469.2009.01170.x

Bolen, R. M., \& Lamb, J. L. (2004). Ambivalence of nonoffending guardians after child sexual abuse disclosure. Journal of Interpersonal Violence, 19(2), 185-211. doi:10.1177/0886260503260324

Campis, L. B., Hebden-Curtis, J., \& DeMaso, D. R. (1993). Developmental differences in detection and disclosure of sexual abuse. Journal of the American Academy of Child and Adolescent Psychiatry, 32(5), 920-924. doi:10.1097/00004583-199309000-00005

Cobb, S. (1976). Social support as a moderator of life stress. Psychosomatic Medicine, 38(5), 300-314.

Collings, S. J., Griffiths, S., \& Kumalo, M. (2005). Patterns of disclosure in child sexual abuse. South African Journal of Psychology, 35(2), 270-285. doi:10.1177/008124630503500207

Conselho Nacional de Saúde. (2012). Resolução $n^{o}$ 466, de 12 de dezembro de 2012. Brasília, DF: Autor. Recuperado em http://www.conselho. saude.gov.br/web comissoes/conep/index.html

Coohey, C., \& O’Leary, P. (2008). Mothers' protection of sexually abused children: An infor- 
mation-processing perspective. Child Abuse \& Neglect: The International Journal, 32(2), 245259. doi:10.1016/j.chiabu.2007.06.002

Crittenden, P. M. (1993). An information processing perspective on the behavior on neglectful behavior. Criminal Justice Behavior, 20(1), 27-48. do i:10.1177/0093854893020001004

Cyr, M., Wright, J., Touopin, J., Oxman-Martinez, J., McDuff, P., \& Theriault, C. (2003). Predictors of maternal support: The point of view of adolescent victims of sexual abuse and their mothers. Journal of Child Sexual Abuse, 12(1), 3965. doi:10.1300/J070v12n01_03

DeYoung, M. (1994). Immediate maternal reactions to the disclosure or discovery of incest. Journal of Family Violence, 9(1), 21-33.doi:10.1007/ BF01531966.

Elliott, A. N., \& Carnes, C. N. (2001). Reactions of nonoffending parents to the sexual abuse of their child: A review of the literature. Child Maltreatment, 6, 314-331.

Everson, M., Hunter, W. N., Runyon, D. K., Edelsohn, M D., \& Coulter, M. L. (1989). Maternal support following disclosure of incest. American Journal of Orthopsychiatry, 59(2), 197-207. doi:10.1111/j.1939-0025.1989.tb01651.x

Faller, K. C. (1988). The myth of the "collusive mother”. Journal of Interpersonal Violence, 3(2), 190-196. doi:10.1177/088626088003002005

Fontes, L. A. (2007). Sin verguenza: Adressing shame with Latino victims of child sexual abuse and their families. Journal of Child Sexual Abuse, 16(1). doi:10.1300/J070v16n01_04.

Heriot, J. (1996). Maternal protectiveness following the disclosure of intrafamilial child sexual abuse. Journal of Interpersonal Violence, 11(2) 181-194. doi:10.1177/088626096011002003

Jonzon, E., \& Lindblad, F. (2004). Disclosure, reactions, and social support: Findings from a sample of adult victims of child sexual abuse. Child Maltreatment, 9(2), 190-200. doi:10.1177/1077559504264263.

Lamb, S., \& Edgar-Smith, S. (1994). Aspects of disclosure: Mediators of outcome of childhood sexual abuse. Journal of Interpersonal Violence, 9(3), 307-326. doi:10.1177/088626094009003002

Lawson, L., \& Chaffin, M. (1992). False negatives in sexual abuse disclosure interviews: Incidence and influence of caretaker's belief in abuse in cases of accidental discovery by diagnosis of STD. Journal of Interpersonal Violence, 7(4), 532-542.

Levenson, J. S., Tewksbury, R., \& DiGiorgio-Miller, J. (2012). Experiences of nonoffending parents and caretakers in child sexual abuse cases. Southwest Journal of Criminal Justice, 8(2), 179-185.

Nagel, D., Putnam, F., Noll, J., \& Trickett, P. (1997). Disclosure patterns of sexual abuse and psychological functioning at a 1-year follow-up. Child Abuse and Neglect, 21(2), 137-147.

Paine, M. L., \& Hansen, D. J. (2002). Factors influencing children to self-disclose sexual abuse. Clinical Psychology Review, 22(2), 271-295. doi:S0272-7358(01)00091-5

Pintello, D., \& Zuravin, S. (2001). Intrafamilial child sexual abuse: Predictors of postdisclosure maternal belief and protective action. Child Maltreatment, 6(4), 344-352.

Plummer, C. A. (2006). The discovery process: What mothers see and do in gaining awareness of the sexual abuse of their children. Child Abuse Neglect, 30(11), 1227-1237. doi:10.1016/j.chiabu.2006.05.007

Programa das Nações Unidas para o Desenvolvimento, Instituto de Pesquisa Econômica Aplicada, \& Fundação João Pinheiro. (2013). Atlas do desenvolvimento humano no Brasil 2013. Recuperado em http://www.atlasbrasil.org.br/2013/

Santos, S. S., \& Dell'Aglio, D. D. (2009). Revelação do abuso sexual infantil: Reações maternas. Psicologia: Teoria e Pesquisa, 25(1), 085-092. doi:10.1590/S0102 37722009000100010

Santos, S. S., \& Dell'Aglio, D. D. (2013). O processo de revelação do abuso sexual na percepção de mães. Revista Psicologia: Teoria e Prática, 15(1), 50-64.

Sarason, I. G., Levine, H. M., Basham, R. B., \& Sarason, B. R. (1983). Assessing social support: The social support questionnaire. Journal of Personality and Social Psychology, 44(1), 127-139.

Sepúlveda, C. C. (2010). Develación del abuso sexual en niños y adolescentes: un articulo de revisión. Revista Chilena de Psiquiatría y Neurología de la Infancia y Adolescencia, 21(1), 44-56.

Sirles, E. A., \& Franke, P. J. (1989). Factors influencing mothers' reactions to intrafamily sexual 
abuse. Child Abuse \& Neglect, 13(1), 131-139. doi:10.1016/0145-2134(89)90036-7

Sgroi, S. M., Blick, L. C., \& Porter, F. S. (1982). A conceptual framework for child sexual abuse. In S. M. Sgroi (Ed.), Handbook of clinical intervention in child sexual abuse (pp. 9-37). Lexington, MA: The Free Press.

Smith, D. W., Letourneau, E. J., Saunders, B. E., Kilpatrick, H. S., Resnick, H. S., \& Best, C. L. (2000). Delay in disclosure of childhood rape: Results from a national survey. Child Abuse and Neglect, 24(2), 273-287. doi:10.1016/S01452134(99)00130-1
Sorensen, T., \& Snow, B. (1991). How children tell: The process of disclosure in child sexual abuse. Child Welfare, 70(1), 3-13. Retrieved from http://www.ncbi.nlm.nih.gov/pubmed/1997290

Tremblay, C., Herbert, M., \& Piche, C. (1999). Coping strategies and social support as mediators of consequences in child sexual abuse victims. Child Abuse \& Neglect, 23, 929-945.

Recebido:18/11/2013

$1^{a}$ revisão: 04/03/2014

Aceite final: $17 / 03 / 2014$

\section{Apêndice}

\section{Entrevista}

A) Questões gerais: enquadramento, revelação, descoberta.

1. Como você ficou sabendo sobre o abuso sexual de(a) seu(sua) filho(a)?

B) Questões de suporte emocional: empatia pelo estresse da criança, ausência ou presença de emoções negativas ou de rejeição.

2. Como você acha que ele(a) se sentiu ao contar-lhe?

3. Porque você acha que o(a) seu(sua) filho(a) levou algum tempo para contar?

4. Você acha que seu(sua) filho(a) poderia ter impedido que o abuso ocorresse?

C) Questões de credibilidade em relação ao relato da criança: acreditar ou não na revelação da vitima.

5. Em algum momento você achou que seu(sua) filho(a) estivesse mentindo? Por quê?

6. O relato do(a) seu(sua) filho(a) foi suficiente? Que tipo de perguntas vocês fez?

7. Que aspecto levou você a ter certeza de que o abuso sexual ocorreu?

D) Respostas comportamentais: ações de proteção, tomada de decisão, etc.

8. O que você fez após a descoberta do abuso sexual?

9. Qual foi a sua atitude em relação ao agressor(a)?

10. Você acha que poderia ter agido de outro modo após a revelação?

E) Percepção sobre a revelação

11. Você conseguiu identificar razões pelas quais o(a) seu(sua) filho(a) contou a você?

12. O que você achou que poderia ter sido feito para manter seu(sua) filho(a) seguro após a revelação?

13. Se ele(ela) não tivesse contado, você acha que descobriria a partir de outros meios?

14. Você consegue citar o que aconteceu de melhor e de pior após a revelação do abuso sexual de(a) seu(sua) filho(a)?

15. Como você descreve o relacionamento com o(a) seu(sua) filho(a) antes e após a revelação ou descoberta do abuso sexual? 